\title{
Avaliação comparativa entre diferentes modalidades de reposição hidroeletrolítica em equinos desidratados experimentalmente pela utilização de parâmetros do exame físico e marcadores hematológicos*
}

\section{Comparative evaluation between different kinds of hydroeletrolitic reposition in horses experimentally dehydrated by the use of physical examination parameters and hematological markers}

Domingos Cachineiro Rodrigues Dias, ${ }^{* *}$ Nadyne Souza Moreira, ${ }^{* * *}$ Thereza Cristina Borio dos Santos Calmon de Bittencourt, ${ }^{* * * *}$ Dyego Pimenta Oliveira, ${ }^{* * * *}$ Pedro Ancelmo Nunes Ermita, ${ }^{* * * * *}$ Micheline Ozana da Silva, ${ }^{* * * * *}$ Leandro Abreu da Fonseca, ${ }^{* * * * * *}$ José Dantas Ribeiro Filho******

\begin{abstract}
Resumo
O objetivo do presente estudo foi realizar a comparação entre três modalidades de terapias hidroeletrolíticas, sendo uma hipotônica (SeHIPO) e outra isotônica (SeISO), ambas por via enteral em fluxo contínuo (HETfc), com a solução de ringer lactato por via intravenosa (RL IV), na taxa de infusão de $15 \mathrm{ml} / \mathrm{kg} / \mathrm{h}$, administradas simultaneamente em tempo real por 8 horas num delineamento crossover 6 X3 em equinos desidratados experimentalmente pela utilização de parâmetros clínicos do exame físico e de alguns exames laboratoriais. Para tanto foram utilizadas 6 éguas adultas da raça Brasileiro de Hipismo submetidas a um protocolo experimental de indução de desidratação (PD) e posteriormente tratadas de acordo com o delineamento experimental. Os resultados obtidos demonstraram que ambos os tratamentos HETfc demonstraram eficácia equivalente ao RL IV na reversão dos efeitos determinados pelo PD, ainda que para os marcadores da volemia, tais como o Volume Globular (VG), Proteínas Plasmáticas Totais (PPT) e porcentagem de Volume Plasmático (\%VP) tenham sido relativamente mais lentos. Porém, para os demais marcadores clínicos a eficácia entre as três terapias estudadas foi equivalente. Esses resultados comprovam a ação das terapias HETfc ao serem comparadas ao tratamento RL IV, pela utilização dos marcadores clínicos e laboratoriais utilizados, e justificam a indicação e utilização dessas modalidades terapêuticas em equinos.
\end{abstract}

Palavras-chave: Equinos, desidratação, hidratação enteral, hidratação intravenosa.

\begin{abstract}
The objective of the present study was to compare three modalities of hydroelectrolytic therapies, one hypotonic (SeHIPO) and another isotonic (SeISO), both by continuous enteral flow (HETfC), with intravenous lactated ringer solution ( RL IV), at the infusion rate of $15 \mathrm{ml} / \mathrm{kg} / \mathrm{h}$, administered simultaneously in real time for 8 hours in a 6X3 crossover design in horses experimentally dehydrated by the use of clinical parameters of the physical examination and some laboratory tests. Six adult mares of the Brazilian Equestrian race were submitted to an experimental protocol for induction of dehydration (PD) and later treated according to the experimental design. The results showed that both HETfc treatments demonstrated an efficacy equivalent to RL IV in the reversal of the effects determined by PD, although for volume markers such as Globular Volume (VG), Total Plasma Proteins (PPT) and Percentage of Plasmatic Volume (\% VP) were relatively slower. However, for the other clinical markers the efficacy among the three therapies studied was equivalent. These results confirm the action of HETfc therapies when compared to RL IV treatment, by the clinical and laboratorial markes used, and justify the indication and use of these therapeutic modalities in horses.
\end{abstract}

Keywords: Equine, dehydration, enteral fluid therapy, intravenous fluid therapy.

\footnotetext{
${ }^{*}$ Recebido em 18 de setembro de 2018 e aceito em 20 de março de 2019.

**Doutorando do Programa de Pós-Graduação em Medicina Veterinária do DVT - UFV, Viçosa-MG e Docente do DEAPAC- EMEVZ - UFBA, Salvador-BA. Autor para correspondência: dcrdias@ufba.br.

***Graduanda de Medicina Veterinária da EMEVZ - UFBA, Salvador-BA.

****Docente do DMVP - EMEVZ - UFBA, Salvador-BA.

*****Docente do Curso de Medicina Veterinária da UNIME, Lauro de Freitas-BA.

***** Doutorando do Programa de Pós-Graduação em Medicina Veterinária do DVT - UFV, Viçosa-MG.

****** Docente do DVT - UFV, Viçosa-MG.
} 


\section{Introdução}

$\mathrm{Na}$ fisiopatogenia das diferentes enfermidades que acometem os equinos levando a significativos prejuízos para a equinocultura destacam-se os diferentes tipos de desequilíbrios hidroeletrolíticos (ALVES et al., 2008), clinicamente reconhecidos como desidratação e/ou hipovolemia, sendo que um cavalo ao ser admitido num serviço médico veterinário invariavelmente estará acometido por diferentes graus de algum desses desequilíbrios (DI FILIPPO et al., 2008).

Tais processos patológicos sempre determinam piora nos quadros clínicos desses pacientes por interferir diretamente na homeostase orgânica e no sucesso das terapias adotadas (LOPES, 2015). A abordagem clínica mais elementar para a avaliação clínica dos desequilíbrios hidroeletrolíticos em equinos são alguns dos parâmetros do exame físico aliados a marcadores laboratoriais da volemia, tais como o volume globular (VG) e as proteínas plasmáticas totais (PPTs) (ALVES et al., 2008). A opção terapêutica de eleição para a correção dos desequilíbrios hídricos e eletrolíticos nos equinos é a hidratação (RIBIERO FILHO, 2009) (LOPES, 2015), onde soluções eletrolíticas formuladas com água, eletrólitos e outros elementos, tais como fontes de energia, são administradas visando o reestabelecimento da volemia, da perfusão sanguínea e, consequentemente, da homeostase geral do equino enfermo. Dentre as diferentes modalidades de terapias hidroeletrolíticas a administração intravenosa da solução de ringer lactato (RL IV) é tida tradicionalmente como a mais eficaz (FIELDING e MAGDESIAN, 2015), sendo a mais utilizada na rotina da medicina equina de forma global (LOPES, 2015).

Porém, já há algum tempo a administração de soluções eletrolíticas por outras vias vem sendo estudada com resultados promissores na espécie equina, tais como a Hidratação Enteral (HET), onde as soluções hidroeletrolíticas são administradas na luz do trato gastrointestinal para então serem absorvidas em direção ao compartimento plasmático, notadamente a HET em Fluxo Contínuo (HETfc) (RIBEIRO FILHO, 2009), com a utilização de diferentes formulações de soluções hidroeletrolíticas administradas por sondas nasoesofágicas de fino calibre, em diversas taxas de infusões contínuas. Esses estudos puderam demonstrar que a HETfc possui diversas vantagens em relação ao tratamento $R L$ IV, tais como seu reduzido custo, maior conforto e menor estresse para o paciente, além da menor incidência de complicações iatrogênicas e a eficácia na correção de desidratações consideradas de leve a moderadas (AVANZA et al., 2009) (GOMES et al., 2012) (PESSIN et al., 2013) (RIBEIRO FILHO et al., 2014a) (RIBEIRO FILHO et al., 2014b) (RIBEIRO FILHO et al., 2015) (RIBEIRO FILHO et al., 2017) que abrangem a maioria dos casos de rotina (ALVES et al., 2008).

Ainda que alguns estudos já tenham demonstrado algumas das vantagens e a eficácia da HETfc frente à terapia RL IV em equinos (LOPES et al., 2002) (RIBEIRO FILHO et al., 2011) (RIBEIRO FILHO et al, 2012) (LESTER et al., 2013) (GOMES et al., 2014) em diferentes delineamentos experimentais, ela ainda permanece como uma modalidade terapêutica restrita a alguns centros, tendo sua eficácia questionada (LOPES, 2015).

Por outro lado, já foi considerado há mais de uma década que a HETfc deve ser a primeira escolha de terapia hidroeletrolítica em equinos (LOPES et al, 2004) devido as suas diversas vantagens, deixando a terapia RL IV para os casos onde e HETfc seria contraindicada, tais como nas situações envolvendo refluxo gastroentérico, atonias intestinais e choque hipovolêmico. Contudo, até o presente momento, ainda não foi realizado um estudo comparativo entre diferentes formulações da HETfc e a terapia RL IV em tempo real e taxas de infusão equivalentes em equinos acometidos por desequilíbrio hidroeletrolítico experimentalmente controlado.

Dessa forma, o presente estudo objetivou realizar a comparação entre duas formulações de tratamento HETfc, sendo uma hipotônica (SeHIPO), uma isotônica (SeISO) e a terapia RL IV, todos em tempo real e na mesma taxa de infusão, utilizandose para esse fim alguns parâmetros clínicos do exame físico, além de marcadores hematológicos da volemia em equinos submetidos a um protocolo experimental de desidratação (PD).

\section{Material e métodos}

Para a realização desse estudo foram utilizados seis equinos hígidos, do sexo feminino, não gestantes, da raça Brasileiro de Hipismo $(\mathrm{BH})$, com idades variando entre 10 e 15 anos, pertencentes ao plantel do Esquadrão de Polícia Montada da Polícia Militar da Bahia (EsquadPMont. - PMBA), identificados aleatoriamente. O delineamento experimental utilizado foi o crossover $6 \mathrm{X} 3$, onde cada animal é submetido em sistema de rodízio a um dos três tratamentos. Cada um dos ciclos experimentais consistiu da aplicação prévia de um Protocolo Experimental de Desidratação (PD) a cada um dos três animais pertencentes àquele ciclo, de acordo com o delineamento proposto.

O PD foi realizado pela aplicação de 36 horas de jejum hídrico e alimentar associadas a duas administrações intravenosas de $1 \mathrm{mg} / \mathrm{kg}$ do diurético furosemida, sendo uma imediatamente no início e outra 12 horas após o início do PD.

Os tratamentos utilizados foram uma solução hipotônica por via HETfc (SeHIPO), uma solução isotônica por via HETfc (SelSO) e a solução de Ringer Lactato por via Intravenosa (RL IV), todos na taxa de infusão de $15 \mathrm{ml} / \mathrm{kg} / \mathrm{h}$ por 8 horas consecutivas. A tabela 1 demonstra a formulação das soluções HETfc. A solução RL utilizada foi a da marca Fresenius $\mathrm{Kabi}^{\circledR}$ contendo $6 \mathrm{~g}$ de cloreto de sódio, 0,27 a 0,4 g de cloreto de potássio, 0,18 a 0,29 g de cloreto de cálcio di-hidratado e 2,3 a 3,3 g de lactato de sódio por litro. Dessa forma cada ciclo experimental era iniciado com o início do PD seguido de imediata administração de um dos três tratamentos de acordo com o delineamento experimental utilizado, como demonstra a tabela 2. Os tempos para realização dos parâmetros do exame físico, assim como a colheita de amostras para os exames laboratoriais hematológicos foram imediatamente antes do início do PD e das aplicações de furosemida (T-36), imediatamente após o final do PD e antes do início dos tratamentos (T0) e duas (T2), quatro (T4), seis (T6) e oito (T8) respectivamente após o início dos tratamentos. Os parâmetros do exame físico foram realizados segundos técnicas de rotina (SPEIRS, 1997). Para essas análises hematológicas as amostras de sangue foram obtidas por venopunção jugular, utilizando agulha e frascos Vacutainer $\mathrm{BD}^{\circledR}$ com anticoagulante (EDTA). Foi utilizada a técnica de micro-hematócrito para confirmação do Volume Globular (VG) mediante a utilização da 
microcentrífuga hematológica Microhemato 2410 Fanem $^{\circledR}$. Para determinação das Proteínas Plasmáticas Totais (PPT) o plasma foi submetido à refratometria no Refratômetro - modelo 8494 (JAIN, 1993). A porcentagem de Volume Plasmático foi calculada pela seguinte fórmula:

$$
\text { \%Volume Plasmático (VP) }=[(\text { PP1/PP2) - 1] } 100
$$

Onde: PP1 é a Proteína Plasmática inicial no T-36 e PP2 é a Proteína Plasmática de cada tempo experimental subjacente (BOYD, 1981).

Tabela 1: Composição (/L) das soluções eletrolíticas administradas por via HETfC

\begin{tabular}{|c|c|c|}
\hline Componentes & $\begin{array}{l}\text { Solução eletrolítica } \\
\text { enteral hipotônica }\end{array}$ & $\begin{array}{c}\text { Solução eletrolítica } \\
\text { enteral isotônica }\end{array}$ \\
\hline Cloreto de Sódio ( $\mathrm{NaCl})$ & $4 g$ & $5 g$ \\
\hline Cloreto de Potássio (KCl) & $0,5 \mathrm{~g}$ & $0,5 \mathrm{~g}$ \\
\hline Acetato de Cálcio $\left(\mathrm{C}_{4} \mathrm{H}_{6} \mathrm{O}_{4} \mathrm{Ca}\right)$ & $1 \mathrm{~g}$ & $1 \mathrm{~g}$ \\
\hline Cloreto de Magnésio $\left(\mathrm{MgCl}_{2}\right)$ & $0,2 \mathrm{~g}$ & $0,2 \mathrm{~g}$ \\
\hline Dextrose $\left(\mathrm{C}_{6} \mathrm{H}_{12} \mathrm{O}_{6}\right)$ & - & $10 \mathrm{~g}$ \\
\hline Maltodextrina $\left(\mathrm{C}_{6 n} \mathrm{H}_{(10 n+2)} \mathrm{O}_{(5 n+1)}\right)$ & $5 \mathrm{~g}$ & - \\
\hline Osmolaridade & $190 \mathrm{mOsm} / \mathrm{L}$ & $280 \mathrm{mOsm} / \mathrm{L}$ \\
\hline
\end{tabular}

Tabela 2: Delineamento experimental crossover $6 \times 3$

\begin{tabular}{cccc}
\hline Ciclo Experimental & SeHIPO & SelSO & RL IV \\
\hline $1^{\circ}$ & Animal 1 & Animal 2 & Animal 3 \\
\hdashline $2^{\circ}$ & Animal 4 & Animal 5 & Animal 6 \\
\hline $3^{\circ}$ & Animal 3 & Animal 1 & Animal 2 \\
\hdashline $4^{\circ}$ & Animal 6 & Animal 4 & Animal 5 \\
\hdashline $5^{\circ}$ & Animal 2 & Animal 3 & Animal 1 \\
\hdashline $6^{\circ}$ & Animal 5 & Animal 6 & Animal 4 \\
\hline
\end{tabular}

Os resultados foram analisados por meio do programa estatístico SPSS 13.0. As variáveis quantitativas foram submetidas aos testes de Normalidade (Shapiro-wilk) e Homocedasticidade (Cochran) e constatado que os pressupostos para o uso da ANOVA foram atendidos. Caso a distribuição da variável fosse normal e a homocesticidade fosse comprovada, foi utilizada análise de variância baseada em planejamento de medidas repetidas para avaliação do efeito dos tratamentos, ou seja, cada tratamento nos diversos vários tempos. Foi analisada também a influência do tempo e a interação entre o tratamento e o tempo. Quando na análise houve efeito significativo para um ou mais fatores foi utilizado o teste de Tukey para comparar todo e qualquer contraste entre duas médias de tratamentos. Todas as análises foram interpretadas considerando o nível de significância de 5\%. Caso a normalidade não fosse comprovada, as diferenças entre tratamentos em um mesmo período foram avaliadas pelo teste de Kruskal-Wallis e as diferenças dentro de tratamento nos diferentes tempos pelo teste de Friedman.
Esse delineamento experimental foi submetido e aprovado pela Comissão de Ética no Uso de Animais da Universidade Federal de Viçosa (UFV) sob o número de protocolo 05/2016.

\section{Resultados e discussão}

A tabela 3 demonstra os resultados relativos ao exame físico dos animais nos diferentes grupos experimentais. Não houve diferenças entre os grupos relacionados aos tratamentos, ainda que em todos os grupos o PD tenha ocasionado aumento do tempo de preenchimento capilar (TPC), demonstrando assim que houve efeito significativo do jejum sobre esse marcador de perfusão tecidual relacionado à desidratação e diminuição da volemia.

A tabela 4 demonstra os resultados para o contorno abdominal e o peso corporal dos animais ao longo do período do estudo. Foi possível notar que o jejum teve efeito significativo sobre esses parâmetros levando à diminuição tanto do contorno abdominal, quanto do peso corporal dos animais.

Em relação ao contorno abdominal foi possível demonstrar que todos os tratamentos foram capazes ao longo do tempo de recuperar os valores perdidos pelo jejum, porém, nos grupos tratados com as soluções enterais, tanto SeHIPO, quanto SelSO, o contorno abdominal a partir das 6 horas de tratamento foi significativamente superior ao grupo tratado com a solução de ringer lactato intravenoso.

Do mesmo modo, o peso corporal dos animais foi significativamente afetado pelo período de jejum, com perda significativa dos seus valores, sendo que todos os tratamentos foram capazes de reverter essa perda de peso corporal, ainda que ao longo dos diferentes tratamentos hidroeletrolíticos os animais estivessem sem acesso à alimentação sólida.

A tabela 5 expõe os valores de parâmetros hematológicos dos animais nos diferentes grupos experimentais. Os valores de volume globular (VG) e de proteínas plasmáticas totais (PPT) foram significativamente afetados pelo PD com aumento dos seus valores, o que caracterizou o desenvolvimento de hemoconcentração. No mesmo sentido, e corroborando esses resultados, os valores de volume plasmático (VP) demonstraram que o jejum foi capaz de levar à diminuição da volemia. Observando a dinâmica desses valores ao longo dos diferentes tratamentos foi possível constatar que o tratamento com Ringer lactato intravenoso demonstrou efeito mais rápido e mais eficaz na recuperação do volume plasmático, tendo levado ao final das 8 horas de tratamento a valores significativamente menores quando comparados ao seu valor inicial e também em relação aos grupos tratados com as soluções enterais, o que caracterizou o desenvolvimento de hemodiluição. Não obstante, foi possível demonstrar que os tratamentos hidroeletrolíticos enterais também foram eficazes em reverter os valores desses três marcadores de volemia, ainda que mais vagarosamente. É importante destacar que, ao contrário do grupo tratado com solução intravenosa, os tratamentos enterais não causaram hemodiluição ao final dos períodos experimentais, tendo tão somente retornado os valores aos seus níveis iniciais. 


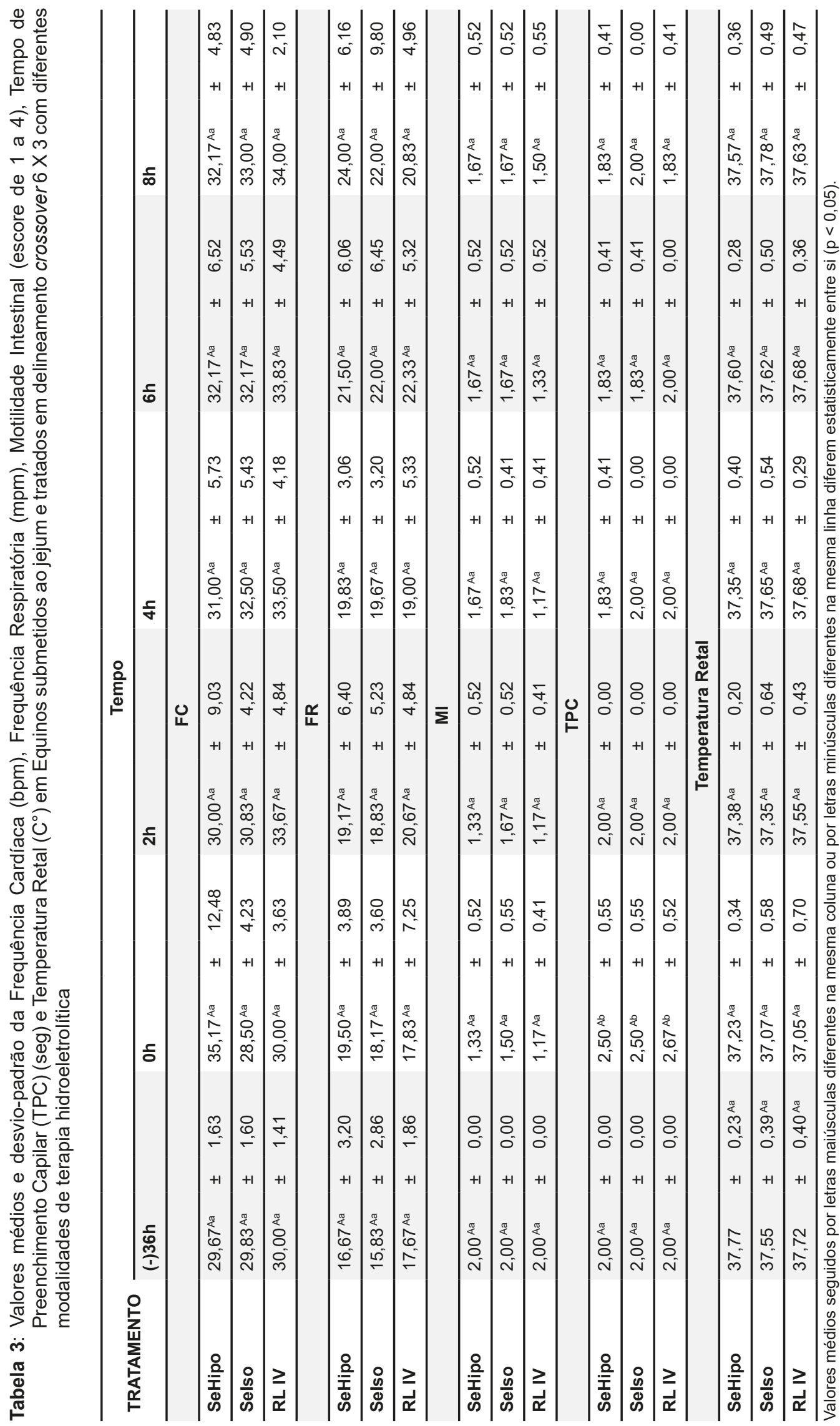




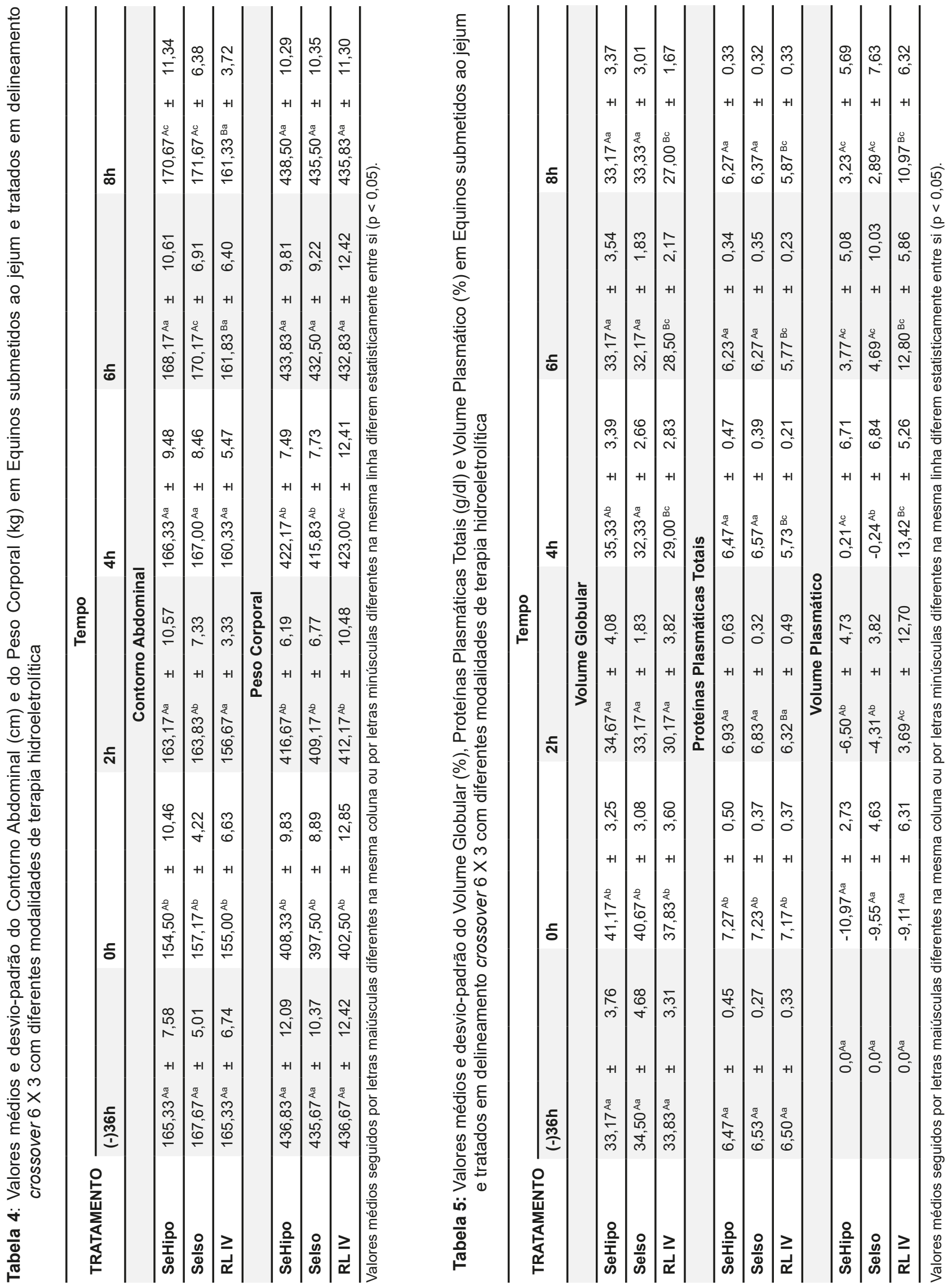


O exame físico é parte fundamental da avaliação do paciente equino acometido de distúrbios dos equilíbrios hídricos e eletrolíticos (ALVES et al., 2008). No presente estudo foram utilizados parâmetros clínicos relacionados ao equilíbrio hidroeletrolítico, tanto do exame físico quanto de exames laboratoriais, para fins de comparação dos tratamentos propostos.

O jejum de 36 horas, associado às administrações de furosemida, representados pelo período $\mathrm{PD}$, apesar de não ter determinado alterações expressivas na maioria dos critérios utilizados no exame físico dos animais utilizados no presente experimento, alterou de forma significativa o Tempo de Preenchimento Capilar (TPC), que pode ser considerado um dos parâmetros mais sensíveis do exame físico para a avaliação do equilíbrio hídrico nos equinos (MELO et al., 2010). O aumento do TPC pode ser traduzido como diminuição da perfusão tecidual periférica ocasionada pelo desenvolvimento de diminuição do volume plasmático circulante (ALVES et al., 2008), sendo que todos os tratamentos propostos foram efetivos em reverter tal efeito. Sendo assim, os tratamentos HETfc tiveram desempenho equivalente ao tratamento RL IV em promover a diminuição do TPC, o seja, pode ser considerado que todos os tratamentos mostraram eficácia em repor a volemia e a perfusão tecidual.

Tal fato foi observado por Silveira et al. (2012), que utilizaram o TPC em equinos como parâmetro de avaliação em animais submetidos a 24 horas de jejum e duas aplicações de furosemida com 12 horas de intervalo e observaram o aumento do TPC em cerca de um quarto dos animais utilizados, creditando tal fato ao desenvolvimento da desidratação. Os autores reportaram o retorno desses valores para a faixa de normalidade após seis horas de tratamento com soluções enterais na mesma taxa de infusão aqui utilizada.

A não observação de alteração nos valores de Frequência Cardíaca, Frequência Respiratória e Temperatura Retal também foi encontrada em estudos que submeteram equinos ao jejum e aplicações de furosemida (AVANZA, 2007) (SILVEIRA et al., 2012) e pode ser justificada pela relativa baixa sensibilidade desses parâmetros do exame físico no monitoramento clínico das alterações advindas da desidratação (ALVES et al., 2008), ainda que a desidratação aqui considerada moderada (MELO et al., 2010), tenha se desenvolvido. No entanto, ao contrário do observado no presente estudo, Silveira et al. (2012) relataram diminuição da motilidade intestinal em equinos advinda do protocolo de 24 horas de jejum e duas aplicações de furosemida que foi revertida após algumas horas de terapia hidroeletrolítica enteral pelo efeito pró-cinético desse tratamento.

O contorno abdominal e o peso dos animais comportaram-se de maneira semelhante, diminuindo de forma significativa seus valores após o período de jejum e aplicações de furosemida. A perda de peso era esperada tanto pelo jejum hídrico, quanto pelo jejum alimentar e foi reportada e em diferentes graus em estudos que utilizaram diferentes protocolos de restrição hídrica e alimentar (AVANZA, 2007) (RIBEIRO FILHO et al., 2012), (SILVEIRA et al., 2012). A diminuição do contorno abdominal justifica-se pela própria perda de peso corporal aliada à diminuição do volume hídrico circulante, recrutamento das reservas de água e eletrólitos intestinais para o compartimento vascular, com consequente diminuição do diâmetro da luz intestinal, notadamente do cólon maior e do ceco, e retração do abdômen, sendo tal fenômeno reportado em experimentos com diferentes protocolos de jejum associados à administração de furosemida (AVANZA, 2007) (RIBEIRO FILHO et al., 2012), (SILVEIRA et al., 2012).

A reposição hídrica em todos os tratamentos foi capaz de reverter a diminuição, do peso corporal, assim como a do contorno abdominal, não havendo diferenças significativas nesses parâmetros entre os dois tratamentos HETfC e RL IV, demonstrando assim que, independente da via de administração utilizada, há reposição dos volumes perdidos e reestabelecimento do ciclo êntero-sistêmico de água entre os compartimentos enterais e o compartimento vascular (WHITE II, 2006).

Contudo, em relação ao contorno abdominal foi possível observar que nas terapias HETfc houve aumento significativo desse parâmetro quando comparadas ao tratamento RL IV nos momentos finais dos períodos experimentais, fato esse creditado ao acúmulo de líquidos nas vísceras abdominais. De acordo com Ribeiro Filho (2009) e Lopes (2015), uma das principais restrições ao uso da hidratação enteral nos equinos é a possibilidade da ocorrência de distensão e desconforto abdominal, podendo até haver ectopias de alças de ceco e cólon maior induzidas pelas terapias HETfc. Porém, em outros estudos utilizando terapias HET nessa espécie, com ou sem períodos prévios de jejum total ou restrição parcial hídrica e alimentar, associadas ou não ao uso de diuréticos, o aumento do contorno abdominal foi observado, sem terem sido observadas tais complicações (RIBEIRO FILHO et al., 2017), (RIBEIRO FILHO et al., 2015), inclusive em protocolos experimentais de indução de compactações de cólon maior (RIBEIRO FILHO et al., 2012), assim como no presente estudo onde, apesar da diferença determinada para os valores de contorno abdominal nos grupos tratados com soluções enterais, não ter sido observado em momento algum desconforto abdominal em nenhum animal tratado com as soluções enterais.

Tal fato corrobora a capacidade absortiva de água dos segmentos intestinais dos equinos, notadamente o ceco e os segmentos do cólon maior (WHITE II, 2006). Ainda que se possa argumentar que os animais do presente estudo sejam animais hígidos, Lopes (2015) afirmou que em situações de processos inflamatórios tais como tiflites e colites que determinam quadros diarreicos em equinos as terapias HET ainda são indicadas, uma vez que as funções de absorção permanecem preservadas a despeito da inflamação.

Aliados aos parâmetros do exame físico, as variáveis laboratoriais hematológicas, tais como o volume globular (VG), juntamente com as proteínas plasmáticas totais (PPT) são marcadores da volemia (ALVES et al., 2008). A dinâmica desses valores confirmou o desenvolvimento de diminuição da volemia e hemoconcentração pelo aumento significativo de seus valores após o PD. Tal fato era esperado e foi observado em estudos que utilizaram outros protocolos de restrição hídrica (RIBEIRO FILHO et al., 2003) (AVANZA, et al., 2009) (SILVEIRA et al., 2012). Os três tratamentos foram capazes de reduzir os valores de VG e PPT de forma significativa ao longo do tempo, fato que confirma a promoção da expansão e da reposição do volume plasmático. A dinâmica dos marcadores hematológicos da volemia atestam a eficácia dos tratamentos HETfc na promoção da volemia, ainda que de forma mais vagarosa, já que nessa 
modalidade terapêutica as soluções precisam ter tempo para serem transportadas do estômago e do intestino delgado e absorvidas pelo ceco e segmentos do cólon maior (LOPES, 2015). Porém, o tratamento RL IV manteve esses valores significativamente menores em relação aos tratamentos HETfc já a partir das seis horas de tratamento.

Essa eficácia do tratamento RL IV em promover a volemia é consagrada (FIELDING e MAGDESIAN, 2015). Por outro lado, apesar de poderem ser consideradas inferiores nesse sentido em relação à terapia intravenosa (LOPES, 2015), a ação eficaz das terapias HETfc em promover a volemia pela utilização do VG e das PPT como parâmetros laboratoriais já foi reportada (RAINGER e DART, 2006) (RIBEIRO FILHO, 2009) (LOPES, 2015).

Contudo, no presente estudo foi demonstrada a diferença entre a resposta desses parâmetros laboratoriais da volemia de acordo com os tratamentos hídricos utilizados. Dessa forma, foi possível comprovar que já nas horas iniciais as terapias HETfc foram menos eficientes quanto o tratamento RL IV na promoção do volume plasmático, confirmando que a terapia intravenosa é mais veloz para essa reposição.

Porém, é preciso ser destacado que o VG, assim como as $\mathrm{PPT}$, como marcadores da volemia e do equilíbrio hídrico são parâmetros sujeitos a diversos fatores fisiológicos que determinam variações não determinadas pelo equilíbrio hídrico, fato que limita a sensibilidade dessas variáveis laboratoriais na avaliação do perfil hídrico dos equinos (WALTON, 2013) (McGOWAN e HODGSON, 2014).

Os valores de porcentagem de volume plasmático demonstraram que o grau de desidratação desenvolvido pelo protocolo prétratamento foi de cerca de $10 \%$, o que é considerada uma desidratação moderada (ALVES et al., 2008). A dinâmica desses valores corroborou os resultados observados para o VG e as

\section{Referências}

ALVES, G.E.S.; BOTTEON, P.T.L.; RIBEIRO FILHO, J.D.; OLIVEIRA, H.P. Fluidoterapia em equinos. Revista Brasileira de Medicina Equina, v. 18, p. 14-24, 2008.

AVANZA, M.F.B. Soluções eletrolíticas associadas ou não a glicose, maltodextrina, e sulfato de magnésio administrada por via enteral em equinos hígidos e desidratados experimentalmente. 2007. 68 f. Dissertação (Mestrado em Medicina Veterinária) Departamento de Veterinária, Universidade Federal de Viçosa, Viçosa, MG, 2007.

AVANZA, M.F.B.; RIBEIRO FILHO, J.D.; LOPES, M.A.F.; IGNÁCIO, F.S.; CARVALHO, T.A.; GUIMARÃES, J.D. Hidratação enteral em equinos - solução eletrolítica associada ou não à glicose, à maltodextrina e ao sulfato de magnésio: resultados de laboratório. Ciência Rural, v. 39, n. 4, p. 1116-1123, 2009.

BOYD, J. W. The relationships between blood haemoglobin concentration, packed cell volume and plasma protein concentration in dehydration. The British Veterinary Journal, v.137, p. 166-172, 1981.

DI FILIPPO, P.A.; SANTANA, A.E.; PEREIRA, G.T. Equilíbrio ácido base e hidroeletrolítico em equinos com cólica. Ciência Rural, v. 38, n. 4, 2008.
PPT onde o tratamento RL por via intravenosa foi mais rápido na promoção da expansão plasmática e do volume vascular.

Por outro lado, o efeito da terapia RL IV em aumentar progressivamente o volume plasmático, devido à velocidade de infusão e sua composição eletrolítica, pode caracterizar uma desvantagem dessa terapia, principalmente na fase de manutenção de um plano terapêutico de hidratação para o paciente equino, uma vez que esse rápido aumento do volume plasmático estimula mecanismos endócrinos regulatórios da volemia promovendo aumento da TFG e micção excessiva de urina hipostenúrica, dificultando assim que o volume administrado no leito vascular seja absorvido para os componentes intersticiais e intracelulares.

\section{Conclusões}

A terapia com a solução de ringer lactato por via intravenosa apresentou mais rapidez na promoção da reposição volêmica, fato que se caracteriza como uma vantagem dessa terapia, principalmente na fase de reposição de um plano terapêutico de hidratação em equinos e em situações de desidratação e hipovolemia intensas nesses animais.

Por outro lado, as terapias HETfc, além de também terem sido eficazes em repor a volemia, já que ao final das 8 horas de tratamento reverteram os efeitos do PD sobre os marcadores hematológicos da volemia, ainda que de forma mais vagarosa, demonstram segurança, uma vez que não houve complicações tais como distensão e desconforto abdominal. Dessa forma, foi possível comprovar a eficácia das terapias HETfc frente a terapia RL IV na reversão dos efeitos do PD pela utilização dos parâmetros clínicos e laboratoriais utilizados. Tal fato corrobora a indicação dessas modalidades terapêuticas na rotina clínica da Medicina Equina.

FIELDING, C.L.; MAGDESIAN, K.G. (Eds.) Equine Fluid Theray, Oxford: Wiley Blackwell, 2015, 374 p.

GOMES, C. L. N., RIBEIRO FILHO, J. D., GUIMARÃES, J. D., MENESES, R. M. Efeitos do polietilenoglicol 3350 e de soluções acidificantes e alcalinizantes sobre o perfil bioquímico de éguas ígidas. Arquivo Brasileiro de Medicina Veterinária e Zootecnia, v. 64, n. 4, p. 817-826, 2012.

GOMES, C.L.N; RIBEIRO FILHO, J.D.; FARIAS, S.K.; DONNER A.C. Efeitos do PEG 3350 e de uma solução enteral, associados ou não ao ringer lactato, e do $\mathrm{NaCl} 0,9 \%$ sobre a glicose, o lactato, o cortisol e a insulina de equinos hígidos. Arquivos Brasileiros de Medicina Veterinária e Zootecnia, v. 66, n.4, p. 1139 - 1145, 2014

JAIN, N.C. Essentials of Veterinary Hematology. 1. ed., Lea e Febiger Eds., Philadelphia, 1993, 417 p.

LESTER, G.D.; MERRITT, A.M.; KUCK, H.V.; BURROW, J.A. Systemic, renal, and colonic effects of intravenous and enteral rehydration in horses. Journal of Veterinary Internal Medicine, v. 27, p. 544-566, 2013.

LOPES, M. A. F.; WALKER, B.L.; WHITE II, N.A.; WARD, D.L. Treatments to promote colonic hydration: enteral fluid therapy versus intravenous fluid therapy and magnesium sulphate. Equine Veterinary Journal, v. 34, n. 5, p. 505-509, 2002. 
LOPES, M.A.F.; MAIA, L.; AVANZA, M.F.B.; COSTA, E.S.; BITTENCOURT VAGO, P.; FLAUSINO, A.B.; LEDNICK., A.C. Fluidoterapia enteral em cavalos: relatos de casos clínicos e cirúrgicos. Brazilian Journal of Veterinary Research and Animal Science, v. 41 (supl), p. 270-271, 2004.

LOPES, M.A.F. Enteral fluid therapy IN: FIELDING, C.L.; MAGDESIAN, K.G. (Eds.) Equine Fluid Therapy, Oxford: Wiley Blackwell, 2015, p. $261-278$.

MCGOWAN, C.M.; HODGSON, D.R. Hematology and biochemistry. IN: HODGSON, D.R..; MCKEEVER, K.H.; MCGOWAN, C.M. The athletic horse: principles and practice of equine sports medicine. Elsevier Health Sciences, 2014.

MELO, U.P.; FERREIRA, C.; PALHARES, M.S.; SILVA FILHO, J.P. Choque circulatório em equinos. Semina: Ciências Agrárias, V. 31, n. 1, 2010.

PESSIN, A.E., RIBEIRO FILHO, J.D.; DONNER, A.C.; OLIVEIRA, D.P.; LIMA, A.P.; FONSECA, B.P.A. Soluções eletrolíticas enterais hipotônicas em equinos: efeitos de fontes de energia sobre determinados indicadores do equilíbrio ácido base. Ciência Rural, V. 43, n. 12, 2013.

RAINGER, J. E.; DART, A. J. Enteral fluid therapy in large animals. Australian veterinary journal, v. 84, n. 12, p. 447-451, 2006.

RIBEIRO FILHO, J.D. Hidratação enteral em ruminantes e equídeos. Eficiência com menos custo. Revista do Conselho Federal de Medicina Veterinária, n. 48, p. 63-67, 2009.

RIBEIRO FILHO, J.D.; ALVES, G.E.S.; DANTAS, W.M.F. Tratamentos da compactação experimental do cólon maior de equinos com hidratação enteral, intravenosa e sene (Cassia augustifolia Vahl). Revista Ceres, v. 59, n. 1, 2012.

RIBEIRO FILHO, J.D.; DANTAS, W.M.F.; ALVES, G.E.S. Cortisol sérico de equinos com compactação experimental no cólon maior tratados com hidratações enteral e parenteral. Revista Ceres, v. 58, n.3, p. 288-292, 2011.
RIBEIRO FILHO, J.D.; FARIAS, S.K., FONSECA, L.A.; AVANZA, M.F.B., DANTAS, W.M.F.; DIAS, D.C.R., MONTEIRO, L.C., RIBEIRO, B.M.; DANTAS, F.T.D. Enteral electrolyte solutions with different osmolarities: clinical and laboratory assessment in equines. Journal of Equine Veterinary Science, v. 35, p. 673-678, 2015.

RIBEIRO FILHO, J.D.; FARIAS, S.K.; DONNER, A.C.; OLIVEIRA, D.P.; GUIMARÃES, J.D.; SOUZA, M.V.; GOMES, C.L.N.;

AVANZA, M.F.B. Efeito de soluções eletrolíticas enterais com diferentes osmolaridades sobre o perfil eletrolítico e bioquímico de equinos. Pesquisa Veterinária Brasileira, v. 34, n. 2, p. $179-$ 184, 2014a.

RIBEIRO FILHO, J.D.; PESSIN, A E.; ATOJI, K.; SOUZA, M.V.; GOMES, C.L.N.; SILVA, A.R. Enteral Fluid Therapy: Biochemical profile of horses treated with hypotonic enteral electrolyte solutions associated with energy sources. Journal of Equine Veterinary Science, v. 34, p. 759-764, 2014b.

RIBEIRO FILHO, J.D.; PESSIN, A.E.; FONSECA, L.A.; DANTAS, W.M.F.; COSTA C.M.; ERMITA, P.A.M.; MONTEIRO, L.C.; RIBEIRO, B.M.; SILVA, M.O.; DANTAS, F.T.D.R. Enteral fluid therapy in horses: Effects of maintenance hypotonic electrolyte solutions containing maltodextrin, sucrose, or dextrose administered in continuous flow. Journal of Equine Veterinary Science, v. 50, p. 96-101, 2017.

SILVEIRA, C. O., RIBEIRO FILHO, J. D., DA CRUZ, A. L., MENESES, R. M., DANTAS, W. D. M. F., GUIMARÃES, J. D. Avaliação de equinos submetidos à hidratação enteral com soluções eletrolíticas isotônicas contendo diferentes fontes de energia e cálcio. Archives of Veterinary Science, v. 17, n. 1, 2012.

SPEIRS, Victor C. Clinical examination of horses. Philadelphia, W.B. Saunders, 1997, 358 p.

WALTON, R.M. (Ed.). Equine clinical pathology. John Wiley \& Sons, 2013.

WHITE II, N. A. Equine Colic. Proceedings of the Annual Convention of the AAEP, v. 52, p. 109-174, 2006. 